\title{
Review: PTCA reduces adverse cardiac outcomes and death better than thrombolytics after myocardial infarction
}

\author{
Keeley EC, Boura JA, Grines CL. Primary angioplasty versus intravenous thrombolytic therapy for acute myocardial \\ infarction: a quantitative review of 23 randomised trials. Lancet 2003;361:13-20.

\begin{abstract}
QUESTION: In patients with acute myocardial infarction (MI), is percutaneous transluminal coronary angioplasty (PTCA) more effective than thrombolytic treatment?
\end{abstract}

\section{Data sources}

Studies were identified by searching Medline, reviewing scientific session abstracts in the New England Journal of Medicine and 5 cardiology journals, and contacting authors.

\section{Study selection}

Studies were selected if they were published or unpublished randomised controlled trials (RCTs) comparing primary PTCA with intravenous thrombolytic treatment for acute ST segment MI.

\section{Data extraction}

Data were extracted on patient characteristics, symptom duration, use of stents or glycoprotein IIb/IIIa antagonists, thrombolytic agent used, time to treatment, and results. Outcomes included total mortality, reinfarction, stroke, haemorrhagic stroke, major bleeding, and a combined endpoint of death, reinfarction, and disabling stroke. Short term (4-6 wk) and long term (6-18 mo) effectiveness was assessed.

\section{Main results}

23 trials $(\mathrm{n}=7739)$ met the inclusion criteria. 8 trials compared PTCA with streptokinase $(\mathrm{n}=1837)$ and 15 compared PTCA with fibrin specific agents $(\mathrm{n}=5902)$. Overall, PTCA was superior to thrombolytic treatment in the short term in reducing death, nonfatal reinfarction, stroke, haemorrhagic stroke, and the combined endpoint (table). PTCA was associated with greater risk of major haemorrhage ( $7 \% v 5 \%$, odds ratio $1.30,95 \%$ CI 1.02 to 1.65). The effectiveness of PTCA was maintained during long term follow up. The exclusion from the meta-analysis of 1 trial, which enrolled high risk patients with cardiogenic shock and compared a direct invasive strategy with thrombolysis and intraaortic balloon pump, did not affect the results for all cause mortality (OR 0.70 , CI 0.58 to 0.85 ). The results also were not affected by type of thrombolytic used.

\section{Conclusion}

In patients with acute myocardial infarction, percutaneous transluminal coronary angioplasty is more effective than thrombolytic treatment in reducing adverse cardiac events and death and is less likely to result in haemorrhagic stroke.

\section{COMMENTARY}

RCTs suggest that primary percutaneous coronary intervention (PCI) is a more effective reperfusion strategy than fibrinolysis for the treatment of acute ST segment elevation MI. There is not much debate about the superiority of PCI in reducing reinfarction and stroke rates and hospital length of stay. However, primary PCI is not widely available and substantial time delays in delivery limit the mortality benefit.

Time to treatment and access to expert PCI are critical issues. In the review by Keeley et al, the PCI related time delay in the RCTs was only 40 minutes, but this varied widely from patient to patient and study to study. It is likely that delays are longer in clinical practice in many communities for several logistical reasons. Also, most of the patients receiving PCI were treated by experienced interventionalists in high volume laboratories. Current guidelines state that hospitals should do $\geq 200$ PCI procedures per year, operators should do $\geq 75$ PCI procedures per year, and door to balloon time should be $<120$ minutes. ${ }^{1}$ Otherwise, fibrinolysis is preferred. Hospitals aiming to achieve the same mortality benefit with PCI as that shown in RCTs will have to match their short times to treatment and procedure success rates.

Interhospital transfer for primary PCI, instead of on site fibrinolysis, has recently been promoted by 5 studies, ${ }^{2}$ but the PCI related time delay was 44 minutes, which can only be accomplished in some communities in North America. Primary PCI done at certified hospitals without on site cardiac surgery or initial transport of patients to PCI centres is a better strategy to reduce PCI related time delay. Prehospital fibrinolysis is another mortality reduction strategy and may be preferred in communities where local, expert PCI is not available. The concept that treatment delay up to 3 hours is acceptable for primary PCI instead of fibrinolysis is not supported by current data.

Primary PCI is particularly beneficial in patients who are elderly, have heart failure or cardiogenic shock, or present late. Fibrinolysis with symptom duration $<2$ hours can salvage substantial myocardium with early reperfusion and may be preferable to PCI. Preinterventional laboratory half dose fibrinolysis (facilitated PCI) and rescue PCI for unsuccessful fibrinolysis may offer a superior reperfusion strategy than either fibrinolysis or primary PCI alone.

Eric R Bates, MD

University of Michigan, Ann Arbor, Michigan, USA

1 Ryan TJ, Antman EM, Brooks NH, et al. 1999 update: ACC/AHA guidelines for the management of patients with acute myocardial infarction. A report of the American College Prarce Guilelines (Commitee on Manon Task tore on Practice Guidelines (Committee on Management of Acute

Zilstra F Angioplasty vs thrombolysis for acuse myocardi.

Zilstra F. Angioplasty vs thrombolysis for acute myocardial tal transportation [Editorial]. Eur Heart J 2003;24:21-3.

*Abbreviations defined in glossary; RRR, NNT, and $\mathrm{Cl}$ calculated from data in article using odds ratios. 